\title{
Elements of bi-cubic polynomial natural spline interpolation for scattered data: Boundary conditions meet partition of unity technique
}

\author{
Weizhi $\mathrm{Xu} *$ \\ School of Information Management and Statistics, Hubei University of Economics, China
}

\begin{abstract}
This paper investigates one kind of interpolation for scattered data by bi-cubic polynomial natural spline, in which the integral of square of partial derivative of two orders to $x$ and to $y$ for the interpolating function is minimal (with natural boundary conditions). Firstly, bi-cubic polynomial natural spline interpolations with four kinds of boundary conditions are studied. By the spline function methods of Hilbert space, their solutions are constructed as the sum of bilinear polynomials and piecewise bi-cubic polynomials. Some properties of the solutions are also studied. In fact, bi-cubic natural spline interpolation on a rectangular domain is a generalization of the cubic natural spline interpolation on an interval. Secondly, based on bi-cubic polynomial natural spline interpolations of four kinds of boundary conditions, and using partition of unity technique, a Partition of Unity Interpolation Element Method (PUIEM) for fitting scattered data is proposed. Numerical experiments show that the PUIEM is adaptive and outperforms state-of-the-art competitions, such as the thin plate spline interpolation and the bi-cubic polynomial natural spline interpolations for scattered data.
\end{abstract}

Keywords Scattered Data Interpolation, Partition of Unity, Bi-cubic Polynomial, Natural Spline, Boundary Condition

AMS 2010 subject classifications 41A15; 65D07; 65D17

DOI: $10.19139 /$ soic-2310-5070-1083

\section{Introduction}

In the classical problem of variational spline interpolation one looks for a $C^{2}$ curve $\tilde{s}$ that satisfies the problem

$$
\text { minimize }\left\{\int_{[a, b]}\left|s^{\prime \prime}(t)\right|^{2} d t: s \in S, s\left(t_{i}\right)=y_{i}, i=1,2, \cdots, n\right\},
$$

where $\quad \tau:=\left\{a=t_{1}<\cdots<t_{n}=b\right\} \quad$ is $\quad$ a partition of interval $\quad[a, b], \quad$ and $\quad S:=\left\{s(t): s^{\prime \prime} \in\right.$ $L_{2}[a, b], s^{\alpha}(t)$ are absolutely constinuous on $\left.[a, b], \alpha=0,1\right\}$ is a functional space on $[a, b]$. It is well known that the solution to the above problem is a cubic natural spline that approximate the thin-beam spline of mechanics [16]. The above problem is also called cubic natural spline interpolation for one dimension scattered data[1, 2]. It is also well known that to compute the spline, one solves a linear system of the order of the number of scattered data points within $[a, b]$ that are being interpolated. The global solution has many nice approximate properties.

Though multivariate tensor product B-splines interpolants on gridded data play a significant role in applications, they do not work on scattered data $[3,8,4,6,7]$. The tensor product B-spline representations increase the dimension of the spline space and can not deal directly with multivariate scattered data interpolation. How to construct directly multivariate splines interpolatory basis for scattered data has been yet a challenging work

\footnotetext{
*Correspondence to: Weizhi Xu (Email: xwz0118@hbue.edu.cn). School of Information Management and Statistics, Hubei University of Economics. 8 Yangqiaohu Road, Wuhan, Hubei Province, China (430205).
}

ISSN 2310-5070 (online) ISSN 2311-004X (print)

Copyright (C) 2020 International Academic Press 
$[9,10,5,11,13,17]$. Y. S. Li and L. T. Guan tried to generalize cubic natural spline for deal with univariate scattered data interpolation to bi-cubic natural spline, and studied multivariate optimal interpolation for scattered data problem with continuous boundary conditions and discrete boundary conditions on a rectangular domain in general mixed spline space [9]. C. K. Chui and L. T. Guan generalized the results of bivariate to general multivariate completely [10]. L. T. Guan also studied local supported basis which are similar to B-spline basis. The computing methods for the properties of the local supported basis and interpolating natural spline were published [11]. Because its functional objective is expressed with a series integral terms [9], it is so complicated that it can't be used perfectly. In the paper [13], we focused on the sparest representation of interpolatory basis in truncated powers for scattered data, and studied one kind of interpolation for scattered data by bi-cubic polynomial natural spline, such that the integral of square of partial derivative of two orders to $\mathrm{x}$ and to $\mathrm{y}$ for the interpolating function is minimal (with natural boundary conditions). We constructed its solution and analyzed its approximate properties. Furthermore, our method was easily generalized to multivariate scattered data interpolation.

In this paper, bi-cubic polynomial natural spline interpolations of four kinds of boundary conditions are named four types. We study the types and their respective interpolatory elements. From the properties of interpolatory elements, we conclude that bi-cubic natural spline interpolation on a rectangular domain is a generalization for cubic natural spline interpolation on an interval. The experiments show that the effects of scattered data natural spline fitting in four types are different when the distribution of data is not uniform. Combining partition of unity technique, we propose the Partition of Unity Interpolation Element Method (PUIEM) for fitting scattered data. The experiments show that our PUIEM is adaptive and outperforms the bi-cubic polynomial natural spline interpolations of four kinds and the thin plate spline in the interpolation results for scattered data.

The paper is organized as follows: in Section 2, we recall the problems of bi-cubic natural spline interpolations for scattered data with four kinds of boundary conditions and study their solutions. Section 3 deals with how to solve bi-cubic natural spline interpolations. In Section 4, many properties of bi-cubic natural spline interpolations are given. Section 5 proposes the Partition of Unity Interpolation Element Method (PUIEM) for fitting scattered data and concludes the paper.

\section{Bi-cubic Natural Spline Interpolation for Scattered Data}

Let $\left\{\left(x_{i}, y_{i}, z_{i}\right) \mid i=1,2, \cdots, N\right\}$ be a given scattered data set. Suppose $a<a_{1}<b_{1}<b, c<c_{1}<d_{1}<d$ and consider the rectangular domain $R=\left[a_{1}, b_{1}\right] \times\left[c_{1}, d_{1}\right]$. Let us consider the functional spaces $X=H^{2,2}(R)=$ $\left\{u(x, y) \mid \frac{\partial^{4} u}{\partial x^{2} \partial y^{2}} \in L_{2}(R), \frac{\partial^{\alpha+\beta} u}{\partial x^{\alpha} \partial y^{\beta}}\right.$ are absolutely continuous, $\left.\alpha=0,1, \beta=0,1,(x, y) \in R\right\}$ and $Y=L_{2}(R)$, and the linear operator $T: X \rightarrow Y$ defined as $T(u)=u^{(2,2)}(x, y)=\frac{\partial^{4} u(x, y)}{\partial x^{2} \partial y^{2}}$, with natural boundary conditions as $u^{(2, \nu)}(x, r)=\left.\frac{\partial^{2+\nu} u(x, y)}{\partial x^{2} \partial y^{\nu}}\right|_{y=r}=0, \nu=0,1$. These conditions can also be written as $u^{(2,0)}(x, r)=u^{(2,1)}(x, r)=$ $0, r=c$ or $d$. Similarly, there also exist other natural boundary conditions as follows: $u^{(0,2)}(s, y)=u^{(1,2)}(s, y)=$ $0, s=a$ or $b$.

We denote $Z=\Re^{N}$ the N-dimensional Euclidean space, and we will mark any element in the space by $z=\left(z_{1}, z_{2}, \cdots, z_{N}\right)$. Let $A: X \rightarrow Z$ be the interpolatory operator defined by $A u=\left(u\left(x_{1}, y_{1}\right), \cdots, u\left(x_{N}, y_{N}\right)\right)$.

We study the spline interpolation problem for scattered data in the Hilbert space $H^{2,2}(R)$, which is also called bi-cubic natural spline interpolation problem. Its solution is named bi-cubic natural spline.

Problem: Look for a function $\sigma(x, y) \in X$ such that [12]

$$
\sigma=\arg \min \left\{\|T u\|^{2}: A u=z, u \in X, z=\left(z_{1}, z_{2}, \cdots, z_{N}\right)\right\}
$$

where $\|T u\|^{2}=\iint_{R}\left(u^{(2,2)}(x, y)\right)^{2} \mathrm{~d} x \mathrm{~d} y$ with natural boundary conditions as follows:

$$
\begin{aligned}
& u^{(2,0)}(x, r)=u^{(2,1)}(x, r)=0, r=c \text { or } d, \\
& u^{(0,2)}(s, y)=u^{(1,2)}(s, y)=0, s=a \text { or } b .
\end{aligned}
$$

In practice, there are four kinds of boundary conditions, which are $a c, a d, b c$ and $b d$ as above. In this paper they are named as type I, II, III, and IV respectively. 
Denote by $N(T)$ and $N(A)$ the null spaces of the operator $T$ and $A$ correspondingly, and by $R(T), R(A)$ their ranges. Let $A_{z}:=\{u \in X \mid A u=z, u$ satisfies type I natural boundary conditions $\}$ be a set of $u \in X$ satisfying both interpolatory conditions and type I natural boundary conditions. We consider the existence and uniqueness of the interpolatory solution with type I natural boundary conditions.

It is proved easily the theory as follows:

Theorem 1

The null subspace of the operator $T$ is

$$
N(T)=P\langle 2,2\rangle=\left\{u \mid u(x, y)=c_{00}+c_{10} x+c_{01} y+c_{11} x y\right\} .
$$

The boundary conditions of Theory 2 in [13] is marked by type I, and Theory 2 in [13] is marked by Lemma 1 .

Theorem 2

[12] Let $A_{z} \neq \emptyset$. If the subspace $T N(A)$ is closed in $Y$, then the interpolating natural spline $\sigma$ does exist. Furthermore, if $N(T) \cap N(A)=\left\{\Theta_{X}\right\}\left(\Theta_{X}\right.$ is the null vector in $\left.X\right)$, then the spline $\sigma$ is unique.

\section{Theorem 3}

[12] If $N(T) \cap N(A)=\left\{\Theta_{X}\right\}$, the range $R(T)$ of the operator $T$ is closed in $Y$ and the null space $N(T)$ is finitedimensional, then the subspace $T N(A)$ is closed in $Y$.

\section{Lemma 1}

If $N(T) \cap N(A)=\left\{\Theta_{X}\right\}$, then the solution of bi-cubic natural spline interpolation for scattered data with the boundary conditions of type I does exist and is unique, and its explicit and compactly format expression as follows

$$
\sigma(x, y)=\sum_{i=1}^{N} \lambda_{i} g_{i}(x, y)+c_{00}+c_{10} x+c_{01} y++c_{11} x y,
$$

where $c_{k l}(k=0,1 ; l=0,1)$ and $\lambda_{i}(i=1, \cdots, N)$ are real numbers obtained by the interpolatory conditions. Here $g_{i}(x, y)=G\left(x_{i}, a ; x\right) G\left(y_{i}, c ; y\right)(i=1,2, \cdots, N)$ are bi-cubic natural spline basis where

$$
G\left(r_{i}, t ; \tau\right) \equiv \frac{\left(r_{i}-\tau\right)_{+}^{3}}{3 !}+\frac{\left(r_{i}-t\right)^{2}(\tau-t)}{2 !}-\frac{\left(r_{i}-t\right)^{3}}{3 !}
$$

with $r_{i}=x_{i}, t=a, \tau=x$, or $r_{i}=y_{i}, t=c, \tau=y$.

Theorem 4

If $N(T) \cap N(A)=\left\{\Theta_{X}\right\}$, then the solution of bi-cubic natural spline interpolation for scattered data with the boundary conditions of type IV does exist and is unique, and its explicit and compactly format expression as follows

$$
\sigma(x, y)=\sum_{i=1}^{N} \lambda_{i} g_{i}(x, y)+c_{00}+c_{10} x+c_{01} y+c_{11} x y,
$$

where $c_{k l}(k=0,1 ; l=0,1)$ and $\lambda_{i}(i=1, \cdots, N)$ are real numbers obtained by the interpolatory conditions. Here $g_{i}(x, y)=G_{1}\left(x_{i}, b ; x\right) G_{1}\left(y_{i}, d ; y\right)(i=1,2, \cdots, N)$ are bi-cubic natural spline basis where

$$
G_{1}\left(r_{i}, t ; \tau\right) \equiv \frac{\left(\tau-r_{i}\right)_{+}^{3}}{3 !}+\frac{\left(t-r_{i}\right)^{2}(t-\tau)}{2 !}-\frac{\left(t-r_{i}\right)^{3}}{3 !}
$$

with $r_{i}=x_{i}, t=b, \tau=x$, or $r_{i}=y_{i}, t=d, \tau=y$.

Proof From the theorem 2 and 3, the solution of the bi-cubic natural spline interpolation with the boundary conditions of type IV does exist and is unique. By the spline theory of Hilbert spaces, it is well known that if $\sigma(x, y)$ is the interpolatory spline function solution in Hilbert spaces, then for all $v \in N(T)$, we have $\left\langle T^{*} T \sigma, v\right\rangle=0$, and there exist coefficients $\lambda_{i}$ and $k_{i}$ such that $T^{*} T \sigma=\sum_{i} \lambda_{i} k_{i}$, where $T^{*}$ is the conjugate operate of $T$. Now, the 
results are

$$
T^{*} T \sigma=\sum_{i=1}^{N} \lambda_{i} k_{i} ; \quad \sum_{i=1}^{N} \lambda_{i} x_{i}^{j} y_{i}^{l}=0, j=0,1 ; l=0,1 .
$$

For all $u \in X$, by using Taylor Theorem at $x=b$ firstly, and by using Taylor Theorem at $y=d$ secondly, and from boundary conditions (2) and (4), it is easy to find that

$$
\begin{aligned}
\langle T \sigma, T u\rangle=\sum_{i=1}^{N} \lambda_{i} u\left(x_{i}, y_{i}\right) & =\int_{a}^{b} \int_{c}^{d}\left(\sum_{i=1}^{N} \lambda_{i}\left(\tau-x_{i}\right)_{+}\left(t-y_{i}\right)_{+}\right) u^{(2,2)}(\tau, t) \mathrm{d} t \mathrm{~d} \tau=\langle T \tilde{G}, T u\rangle, \\
\tilde{G}(x, y) & =\sum_{i=1}^{N} \lambda_{i} g_{i}(x, y)+f(x, y), \quad f(x, y) \in N(T),
\end{aligned}
$$

where $g_{i}(x, y)=G_{1}\left(x_{i}, b ; x\right) G_{1}\left(y_{i}, d ; y\right)(i=1,2, \cdots, N)$. Here

$$
\begin{aligned}
& G_{1}^{\prime \prime}\left(x_{i}, b ; x\right)=\frac{\mathrm{d}^{2} G_{1}\left(x_{i}, b ; x\right)}{\mathrm{d} x^{2}}=\left(x-x_{i}\right)_{+}, \\
& G_{1}^{\prime \prime}\left(y_{i}, d ; y\right)=\frac{\mathrm{d}^{2} G_{1}\left(y_{i}, d ; y\right)}{\mathrm{d} y^{2}}=\left(y-y_{i}\right)_{+},
\end{aligned}
$$

and $\tilde{G}(x, y)$ satisfies the boundary conditions (2), thus

$$
g_{i}^{(2,0)}(x, d)=g_{i}^{(2,1)}(x, d)=g_{i}^{(0,2)}(b, y)=g_{i}^{(1,2)}(b, y)=0, i=1, \cdots, N .
$$

So we have

$$
\sigma(x, y)=\sum_{i=1}^{N} \lambda_{i} g_{i}(x, y)+c_{00}+c_{10} x+c_{01} y+c_{11} x y,
$$

where $g_{i}(x, y)=G_{1}\left(x_{i}, b ; x\right) G_{1}\left(y_{i}, d ; y\right)(i=1,2, \cdots, N)$ are bi-cubic natural spline basis and $G_{1}\left(r_{i}, t ; \tau\right) \equiv$ $\frac{\left(\tau-r_{i}\right)_{+}^{3}}{3 !}+c_{0}\left(r_{i}, t\right)+c_{1}\left(r_{i}, t\right) \tau, r_{i}=x_{i}, t=b, \tau=x$, or $r_{i}=y_{i}, t=d, \tau=y$. The coefficients $c_{j}\left(r_{i}, t\right)(j=0,1)$ are obtained by the boundary conditions (5).

Because $g_{i}^{(0,2)}(b, y)=g_{i}^{(1,2)}(b, y)=0$, we get that $\frac{\left(b-x_{i}\right)^{3}}{3 !}+c_{0}\left(x_{i}, b\right)+c_{1}\left(x_{i}, b\right) b=0, \quad$ and $\quad \frac{\left(b-x_{i}\right)^{2}}{2 !}+$ $c_{1}\left(x_{i}, b\right)=0$. Thus $c_{1}\left(x_{i}, b\right)=-\frac{\left(b-x_{i}\right)^{2}}{2 !}$ and $c_{0}\left(x_{i}, b\right)=-\frac{\left(b-x_{i}\right)^{3}}{3 !}+\frac{\left(b-x_{i}\right)^{2}}{2 !} b$. So

$$
\begin{gathered}
G_{1}\left(x_{i}, b ; x\right)=\frac{\left(x-x_{i}\right)_{+}^{3}}{3 !}+c_{0}\left(x_{i}, b\right)+c_{1}\left(x_{i}, b\right) x, \\
c_{0}\left(x_{i}, b\right)=-\frac{\left(b-x_{i}\right)^{3}}{3 !}+\frac{\left(b-x_{i}\right)^{2}}{2 !} b, c_{1}\left(x_{i}, b\right)=-\frac{\left(b-x_{i}\right)^{2}}{2 !} .
\end{gathered}
$$

Similarly, $G_{1}\left(y_{i}, d ; y\right)$ has the same expression as $G_{1}\left(x_{i}, b ; x\right)$. So, we also know that all $g_{i}(x, y)$ for $i=1,2, \cdots, N$ are bi-cubic natural spline basis and

$$
\begin{gathered}
G_{1}\left(r_{i}, t ; \tau\right) \equiv \frac{\left(\tau-r_{i}\right)_{+}^{3}}{3 !}+c_{0}\left(r_{i}, t\right)+c_{1}\left(r_{i}, t\right) \tau, \\
c_{0}\left(r_{i}, t\right)=\frac{\left(t-r_{i}\right)^{2} t}{2 !}-\frac{\left(t-r_{i}\right)^{3}}{3 !}, c_{1}\left(r_{i}, t\right)=-\frac{\left(t-r_{i}\right)^{2}}{2 !},
\end{gathered}
$$

where $r_{i}=x_{i}, t=b, \tau=x$, or $r_{i}=y_{i}, t=d, \tau=y$.

Similarly, as for the boundary conditions of type II there exists the property as follows: 
Theorem 5

If $N(T) \cap N(A)=\left\{\Theta_{X}\right\}$, then the solution of bi-cubic natural spline interpolation for scattered data with the boundary conditions of type II does exist and is unique, and its explicit and compactly format expression as follows

$$
\sigma(x, y)=\sum_{i=1}^{N} \lambda_{i} g_{i}(x, y)+c_{00}+c_{10} x+c_{01} y+c_{11} x y,
$$

where $c_{k l}(k=0,1 ; l=0,1)$ and $\lambda_{i}(i=1, \cdots, N)$ are real numbers obtained by the interpolatory conditions. Here $g_{i}(x, y)=G\left(x_{i}, a ; x\right) G_{1}\left(y_{i}, d ; y\right)(i=1,2, \cdots, N)$ are bi-cubic natural spline basis with type II boundary conditions.

Proof It is similar to the proof of the previous theorem. By using Taylor Theorem at $x=a$ for all $u \in X$ firstly, and secondly by using Taylor Theorem at $y=d$ for $u(a, y), u^{(1,0)}(a, y)$ and $u^{(2,0)}(\tau, y)$.

Similarly, as for the boundary conditions of type III there exists the property as follows:

Theorem 6

If $N(T) \cap N(A)=\left\{\Theta_{X}\right\}$, then the solution of bi-cubic natural spline interpolation for scattered data with the boundary conditions of type III does exist and is unique, and its explicit and compactly format expression as follows

$$
\sigma(x, y)=\sum_{i=1}^{N} \lambda_{i} g_{i}(x, y)+c_{00}+c_{10} x+c_{01} y+c_{11} x y,
$$

where $c_{k l}(k=0,1 ; l=0,1)$ and $\lambda_{i}(i=1, \cdots, N)$ are real numbers obtained by the interpolatory conditions. Here $g_{i}(x, y)=G_{1}\left(x_{i}, b ; x\right) G\left(y_{i}, c ; y\right)(i=1,2, \cdots, N)$ are bi-cubic natural spline basis.

Proof It is similar to the proof of the previous theorem. By using Taylor Theorem at $x=b$ for all $u \in X$ firstly, and secondly by using Taylor Theorem at $y=c$ for $u(b, y), u^{(1,0)}(b, y)$ and $u^{(2,0)}(\tau, y)$.

\section{Resolution of Bi-cubic Natural Spline Interpolation for Scattered Data}

Now, for all given scattered data set $\left\{\left(x_{i}, y_{i}, z_{i}\right) \mid i=1,2, \cdots, N\right\}$, we can fit the scattered data by using bi-cubic natural spline interpolation function as

$$
\sigma(x, y)=\sum_{i=1}^{N} \lambda_{i} g_{i}(x, y)+c_{00}+c_{10} x+c_{01} y+c_{11} x y .
$$

By the interpolatory conditions, $\lambda_{i}(i=1, \cdots, N)$ and $c_{k l}(k=0,1 ; l=0,1)$ can be decided by a linear system as follows

$$
\left(\begin{array}{cc}
A & B \\
B^{T} & 0
\end{array}\right)\left(\begin{array}{c}
\Lambda \\
C
\end{array}\right)=\left(\begin{array}{c}
Z \\
0
\end{array}\right)
$$

where

$$
\begin{gathered}
\Lambda=\left(\lambda_{1}, \lambda_{2}, \cdots, \lambda_{N}\right)^{T}, \quad C=\left(c_{00}, c_{10}, c_{01}, c_{11}\right)^{T}, \quad Z=\left(z_{1}, z_{2}, \cdots, z_{N}\right)^{T} \\
A=\left(a_{i j}\right)_{N \times N}, a_{i j}=g_{i}\left(x_{j}, y_{j}\right), \quad B=(1, x, y, x y)_{N \times 4} .
\end{gathered}
$$

So, they include the interpolation conditions of type I, II, III, and type IV.

\section{Theorem 7}

The coefficient matrix of the linear system (7), which come from bi-cubic natural spline interpolation for scattered data, is symmetric. 
Proof We only prove type II, and the others are similar. It is only needed to prove that $A$ is symmetric. In order to do this, we denote

$$
\begin{gathered}
\alpha_{i j}=\frac{\left(x_{i}-x_{j}\right)_{+}^{3}}{6}-\frac{\left(x_{i}-a\right)^{3}}{6}+\frac{\left(x_{i}-a\right)^{2}\left(x_{j}-a\right)}{2}, \\
\beta_{i j}=\frac{\left(y_{j}-y_{i}\right)_{+}^{3}}{6}-\frac{\left(d-y_{i}\right)^{3}}{6}+\frac{\left(d-y_{i}\right)^{2}\left(d-y_{j}\right)}{2} .
\end{gathered}
$$

Thus, $a_{i j}=\alpha_{i j} \beta_{i j}$.

In the general, suppose $x_{i} \geqslant x_{j}$, then there is

$$
\begin{aligned}
\alpha_{i j} & =\frac{\left(x_{i}-x_{j}\right)^{3}}{6}-\frac{\left(x_{i}-a\right)^{3}}{6}+\frac{\left(x_{i}-a\right)^{2}\left(x_{j}-a\right)}{2} \\
& =\frac{\left[\left(x_{i}-a\right)-\left(x_{j}-a\right)\right]^{3}}{6}-\frac{\left(x_{i}-a\right)^{3}}{6}+\frac{\left(x_{i}-a\right)^{2}\left(x_{j}-a\right)}{2} \\
& =-\frac{\left(x_{j}-a\right)^{3}}{6}+\frac{\left(x_{j}-a\right)^{2}\left(x_{i}-a\right)}{2}=\alpha_{j i} .
\end{aligned}
$$

Suppose $y_{j} \geqslant y_{i}$, then there is

$$
\begin{aligned}
\beta_{i j} & =\frac{\left(y_{j}-y_{i}\right)^{3}}{6}-\frac{\left(d-y_{i}\right)^{3}}{6}+\frac{\left(d-y_{i}\right)^{2}\left(d-y_{j}\right)}{2} \\
& =\frac{\left[\left(d-y_{i}\right)-\left(d-y_{j}\right)\right]^{3}}{6}-\frac{\left(d-y_{i}\right)^{3}}{6}+\frac{\left(d-y_{i}\right)^{2}\left(d-y_{j}\right)}{2} \\
& =-\frac{\left(d-y_{j}\right)^{3}}{6}+\frac{\left(d-y_{j}\right)^{2}\left(d-y_{i}\right)}{2}=\beta_{j i} .
\end{aligned}
$$

So, $a_{i j}=a_{j i}$. This means that $A$ is symmetric.

The interpolatory function is achieved with the values of the coefficients $\lambda_{i}$ and $c_{i j}$ obtained by solving the linear system (7).

\section{Properties of the Bi-cubic Natural Spline Interpolation for Scattered Data}

For all given scattered data set $\left\{\left(x_{i}, y_{i}\right) \mid i=1,2, \cdots, N\right\}$, project them to axis $\mathrm{x}$ and axis $\mathrm{y}$ and get a partition:

$$
\begin{aligned}
& a=x_{0}^{0}<x_{1}^{0}<\cdots<x_{p}^{0}<x_{p+1}^{0}=b, \\
& c=y_{0}^{0}<y_{1}^{0}<\cdots<y_{q}^{0}<y_{q+1}^{0}=d .
\end{aligned}
$$

\section{Theorem 8}

The type I interpolation for scattered data is a bi-linear polynomial on $\left[x_{0}^{0}, x_{1}^{0}\right] \times\left[y_{0}^{0}, y_{1}^{0}\right]$ and $\left[x_{p}^{0}, x_{p+1}^{0}\right] \times\left[y_{q}^{0}, y_{q+1}^{0}\right]$, is a linear polynomial to $y$ and a cubic polynomial to $x$ on $\left[x_{0}^{0}, x_{1}^{0}\right] \times\left[y_{q}^{0}, y_{q+1}^{0}\right]$, and is a linear polynomial to $x$ and a cubic polynomial to $y$ on $\left[x_{p}^{0}, x_{p+1}^{0}\right] \times\left[y_{0}^{0}, y_{1}^{0}\right]$.

Proof The proof will use many times this property:

$$
\sum_{i=1}^{N} \lambda_{i} f\left(x_{i}, y_{i}\right)=0, \forall f(x, y) \in N(T) .
$$




$$
\text { If } \begin{aligned}
&(x, y) \in\left[x_{0}^{0}, x_{1}^{0}\right] \times\left[y_{0}^{0}, y_{1}^{0}\right] \\
& \qquad \begin{aligned}
\sigma(x, y) & =\sum_{i=1}^{N} \lambda_{i}\left(\frac{\left(x_{i}-x\right)_{+}^{3}}{6}-\frac{\left(x_{i}-a\right)^{3}}{6}+\frac{\left(x_{i}-a\right)^{2}(x-a)}{2}\right)\left(\frac{\left(y_{i}-y\right)_{+}^{3}}{6}-\frac{\left(y_{i}-c\right)^{3}}{6}+\frac{\left(y_{i}-c\right)^{2}(y-c)}{2}\right) \\
& +c_{00}+c_{10} x+c_{01} y+c_{11} x y \\
& =\sum_{i=1}^{N} \lambda_{i}\left(\frac{\left(x_{i}-x\right)^{3}}{6}-\frac{\left(x_{i}-a\right)^{3}}{6}+\frac{\left(x_{i}-a\right)^{2}(x-a)}{2}\right)\left(\frac{\left(y_{i}-y\right)^{3}}{6}-\frac{\left(y_{i}-c\right)^{3}}{6}+\frac{\left(y_{i}-c\right)^{2}(y-c)}{2}\right) \\
& +c_{00}+c_{10} x+c_{01} y+c_{11} x y \\
& =\sum_{i=1}^{N} \lambda_{i}\left(-\frac{(x-a)^{3}}{6}+\frac{(x-a)^{2}\left(x_{i}-a\right)}{2}\right)\left(-\frac{(y-c)^{3}}{6}+\frac{(y-c)^{2}\left(y_{i}-c\right)}{2}\right)+c_{00}+c_{10} x+c_{01} y+c_{11} x y \\
& =c_{00}+c_{10} x+c_{01} y+c_{11} x y .
\end{aligned}
\end{aligned}
$$

It is a bi-linear polynomial to $x$ and $y$.

$$
\begin{aligned}
& \text { If }(x, y) \in\left[x_{p}^{0}, x_{p+1}^{0}\right] \times\left[y_{q}^{0}, y_{q+1}^{0}\right] \\
& \begin{aligned}
\sigma(x, y) & =\sum_{i=1}^{N} \lambda_{i}\left(\frac{\left(x_{i}-x\right)_{+}^{3}}{6}-\frac{\left(x_{i}-a\right)^{3}}{6}+\frac{\left(x_{i}-a\right)^{2}(x-a)}{2}\right)\left(\frac{\left(y_{i}-y\right)_{+}^{3}}{6}-\frac{\left(y_{i}-c\right)^{3}}{6}+\frac{\left(y_{i}-c\right)^{2}(y-c)}{2}\right) \\
& +c_{00}+c_{10} x+c_{01} y+c_{11} x y \\
& =\sum_{i=1}^{N} \lambda_{i}\left(-\frac{\left(x_{i}-a\right)^{3}}{6}+\frac{\left(x_{i}-a\right)^{2}(x-a)}{2}\right)\left(-\frac{\left(y_{i}-c\right)^{3}}{6}+\frac{\left(y_{i}-c\right)^{2}(y-c)}{2}\right)+c_{00}+c_{10} x+c_{01} y+c_{11} x y .
\end{aligned}
\end{aligned}
$$

It is also a bi-linear polynomial to $x$ and $y$.

$$
\begin{aligned}
\text { If }(x, y) & \in\left[x_{0}^{0}, x_{1}^{0}\right] \times\left[y_{q}^{0}, y_{q+1}^{0}\right] \\
\sigma(x, y)= & \sum_{i=1}^{N} \lambda_{i}\left(\frac{\left(x_{i}-x\right)_{+}^{3}}{6}-\frac{\left(x_{i}-a\right)^{3}}{6}+\frac{\left(x_{i}-a\right)^{2}(x-a)}{2}\right)\left(\frac{\left(y_{i}-y\right)_{+}^{3}}{6}-\frac{\left(y_{i}-c\right)^{3}}{6}+\frac{\left(y_{i}-c\right)^{2}(y-c)}{2}\right) \\
& +c_{00}+c_{10} x+c_{01} y+c_{11} x y \\
& =\sum_{i=1}^{N} \lambda_{i}\left(\frac{\left(x_{i}-x\right)^{3}}{6}-\frac{\left(x_{i}-a\right)^{3}}{6}+\frac{\left(x_{i}-a\right)^{2}(x-a)}{2}\right)\left(-\frac{\left(y_{i}-c\right)^{3}}{6}+\frac{\left(y_{i}-c\right)^{2}(y-c)}{2}\right) \\
& +c_{00}+c_{10} x+c_{01} y+c_{11} x y \\
= & \sum_{i=1}^{N} \lambda_{i}\left(-\frac{(x-a)^{3}}{6}+\frac{(x-a)^{2}\left(x_{i}-a\right)}{2}\right)\left(-\frac{\left(y_{i}-c\right)^{3}}{6}+\frac{\left(y_{i}-c\right)^{2}(y-c)}{2}\right)+c_{00}+c_{10} x+c_{01} y+c_{11} x y \\
= & (x-a)^{2} \sum_{i=1}^{N} \lambda_{i}\left(-\frac{x-a}{6}+\frac{x_{i}-a}{2}\right)\left(-\frac{\left(y_{i}-c\right)^{3}}{6}+\frac{\left(y_{i}-c\right)^{2}(y-c)}{2}\right)+c_{00}+c_{10} x+c_{01} y+c_{11} x y .
\end{aligned}
$$

It is a linear polynomial to $y$ and a cubic polynomial to $x$. 


$$
\text { If } \begin{aligned}
(x, y) & \in\left[x_{p}^{0}, x_{p+1}^{0}\right] \times\left[y_{0}^{0}, y_{1}^{0}\right] \\
\sigma(x, y) & =\sum_{i=1}^{N} \lambda_{i}\left(\frac{\left(x_{i}-x\right)_{+}^{3}}{6}-\frac{\left(x_{i}-a\right)^{3}}{6}+\frac{\left(x_{i}-a\right)^{2}(x-a)}{2}\right)\left(\frac{\left(y_{i}-y\right)_{+}^{3}}{6}-\frac{\left(y_{i}-c\right)^{3}}{6}+\frac{\left(y_{i}-c\right)^{2}(y-c)}{2}\right) \\
& +c_{00}+c_{10} x+c_{01} y+c_{11} x y \\
& =\sum_{i=1}^{N} \lambda_{i}\left(-\frac{\left(x_{i}-a\right)^{3}}{6}+\frac{\left(x_{i}-a\right)^{2}(x-a)}{2}\right)\left(\frac{\left(y_{i}-y\right)^{3}}{6}-\frac{\left(y_{i}-c\right)^{3}}{6}+\frac{\left(y_{i}-c\right)^{2}(y-c)}{2}\right) \\
& +c_{00}+c_{10} x+c_{01} y+c_{11} x y \\
& =(y-c)^{2} \sum_{i=1}^{N} \lambda_{i}\left(-\frac{\left(x_{i}-a\right)^{3}}{6}+\frac{\left(x_{i}-a\right)^{2}(x-a)}{2}\right)\left(-\frac{y-c}{6}+\frac{y_{i}-c}{2}\right)+c_{00}+c_{10} x+c_{01} y+c_{11} x y .
\end{aligned}
$$

It is a linear polynomial to $x$ and a cubic polynomial to $y$.

We can directly verify that there exist the property as follow:

\section{Theorem 9}

The type I interpolation for scattered data is a linear polynomial to $x$ and a cubic polynomial to $y$ on $\left[x_{p}^{0}, x_{p+1}^{0}\right] \times\left[y_{j}^{0}, y_{j+1}^{0}\right]$, a linear polynomial to $y$ and a cubic polynomial to $x$ on $\left[x_{j}^{0}, x_{j+1}^{0}\right] \times\left[y_{q}^{0}, y_{q+1}^{0}\right]$, and a bi-cubic polynomial to $x$ and $y$ on $\left[x_{0}^{0}, x_{1}^{0}\right] \times\left[y_{j}^{0}, y_{j+1}^{0}\right]$ and $\left[x_{j}^{0}, x_{j+1}^{0}\right] \times\left[y_{0}^{0}, y_{1}^{0}\right]$.

The other types have similar properties, and they also have similar proofs. Theory 6 and Theory 7 show that bi-cubic natural spline interpolation on a rectangular domain is a generalization of the cubic natural spline interpolation on an interval.

Now let $\quad N S:=\left\{s(x, y) \mid s(x, y)=\sum_{i=1}^{N} \lambda_{i} G\left(x_{i}, a ; x\right) G\left(y_{i}, c ; y\right)+\sum_{k=0}^{1} \sum_{l=0}^{1} c_{k l} x^{k} y^{l}\right.$, and $\sum_{i=1}^{N} \lambda_{i} x_{i}^{\mu} y_{i}^{\nu}=0, \mu=$ $0,1, \nu=0,1\}$ be a set of natural bi-cubic spline, and $A_{z}:=\{u \in X \mid A u=z, u$ satisfies type $I$

natural boundary conditions $\}$ be a set of $u \in X$ satisfying both interpolatory conditions and type I natural boundary conditions.

\section{Lemma 2}

Let $u \in X$ satisfy type I natural boundary conditions, and let $\sigma(x, y) \in N S$. Then

$$
\langle T u, T \sigma\rangle=\iint_{R} u^{(2,2)}(x, y) \sigma^{(2,2)}(x, y) \mathrm{d} x \mathrm{~d} y=\sum_{i=1}^{N} \lambda_{i} u\left(x_{i}, y_{i}\right) .
$$

Proof Using integration by parts with respect to $x$, we have

$$
\langle T u, T \sigma\rangle=\iint_{R} u^{(2,2)} \sigma^{(2,2)} \mathrm{d} x \mathrm{~d} y=\int_{c}^{d}\left\{\left.\left[u^{(1,2)} \sigma^{(2,2)}-u^{(0,2)} \sigma^{(3,2)}\right]\right|_{a} ^{b}+\int_{a}^{b} u^{(0,2)} \sigma^{(4,2)} \mathrm{d} x\right\} \mathrm{d} y .
$$

From boundary conditions $u^{(0,2)}(a, y)=u^{(1,2)}(a, y)=0$ and $\sigma \in N S$, we have

$$
\begin{aligned}
& \left.u^{(1,2)} \sigma^{(2,2)}\right|_{a} ^{b}=\left.\left[u^{(1,2)} \sum_{i=1}^{N} \lambda_{i}\left(x_{i}-x\right)_{+}\left(y_{i}-y\right)_{+}\right]\right|_{a} ^{b}=0, \\
& \left.u^{(0,2)} \sigma^{(3,2)}\right|_{a} ^{b}=\left.\left[u^{(0,2)} \sum_{i=1}^{N} \lambda_{i}\left(x_{i}-x\right)_{+}^{0}\left(y_{i}-y\right)_{+}\right]\right|_{a} ^{b}=0 .
\end{aligned}
$$


Thus, $\iint_{R} u^{(2,2)} \sigma^{(2,2)} \mathrm{d} x \mathrm{~d} y=\int_{c}^{d} \int_{a}^{b} u^{(0,2)} \sigma^{(4,2)} \mathrm{d} x \mathrm{~d} y$.

Similarly, using integration by parts with respect to $y$, from type I boundary conditions we have

$$
\begin{aligned}
\langle T u, T \sigma\rangle & =\iint_{R} u^{(2,2)}(x, y) \sigma^{(2,2)}(x, y) \mathrm{d} x \mathrm{~d} y=\int_{c}^{d} \int_{a}^{b} u(x, y) \sigma^{(4,4)}(x, y) \mathrm{d} x \mathrm{~d} y \\
& =\int_{c}^{d} \int_{a}^{b} u(x, y)\left[\sum_{i=1}^{N} \lambda_{i} \delta\left(x_{i}-x\right) \delta\left(y_{i}-y\right)\right] \mathrm{d} x \mathrm{~d} y=\sum_{i=1}^{N} \lambda_{i} u\left(x_{i}, y_{i}\right),
\end{aligned}
$$

where $\delta(x)=\left(x_{+}^{0}\right)^{\prime}$.

Theorem 10 (Minimal Modulus Property)

Suppose that $u \in A_{z}, \sigma \in N S \bigcap A_{z}$, and denote $r=u-\sigma$, then

$$
\|T u\|^{2}=\|T r\|^{2}+\|T \sigma\|^{2} .
$$

Thus $\quad\|T \sigma\|^{2}=\min _{u \in A_{z}}\|T u\|^{2}$.

Proof It follows that

$$
\begin{aligned}
\|\operatorname{Tr}\|^{2} & =\iint_{R}\left(u^{(2,2)}(x, y)-\sigma^{(2,2)}(x, y)\right)^{2} d x d y \\
& =\iint_{R}\left(u^{(2,2)}(x, y)\right)^{2} d x d y-2 \iint_{R}\left(u^{(2,2)}(x, y)-\sigma^{(2,2)}(x, y)\right) \sigma^{(2,2)}(x, y) d x d y \\
& -\iint_{R}\left(\sigma^{(2,2)}(x, y)\right)^{2} d x d y .
\end{aligned}
$$

Because $u\left(x_{i}, y_{i}\right)-\sigma\left(x_{i}, y_{i}\right)=0, i=1,2, \cdots, N$ and the Lemma 2, we have

$$
\iint_{R}\left(u^{(2,2)}(x, y)-\sigma^{(2,2)}(x, y)\right) \sigma^{(2,2)}(x, y) \mathrm{d} x \mathrm{~d} y=0 .
$$

Then

$$
\|\operatorname{Tr}\|^{2}=\iint_{R}\left(u^{(2,2)}(x, y)\right)^{2} d x d y-\iint_{R}\left(\sigma^{(2,2)}(x, y)\right)^{2} d x d y
$$

This means

$$
\|T u\|^{2}=\|T r\|^{2}+\|T \sigma\|^{2} .
$$

From the formula, it follows that

$$
\|T \sigma\|^{2}=\min _{u \in A_{z}}\|T u\|^{2}
$$

Theorem 11 (Best Approximating Property)

Suppose that $u \in A_{z}, \sigma \in N S \bigcap A_{z}$, then for all $s \in N S$, such that

$$
\|T(u-\sigma)\|^{2}=\min _{s \in N S}\|T(u-s)\|^{2} .
$$

If there exists another $\tilde{\sigma}$ satisfies this property, that the difference between $\sigma$ and $\tilde{\sigma}$ is only a bi-linear polynomial. 
Proof Using $u-s$ replace $u$ and $\sigma-s$ replace $\sigma$ in the Theorem 10, it follows that

$$
\|T(u-s)\|^{2}=\|T(u-\sigma)\|^{2}+\|T(\sigma-s)\|^{2} .
$$

This means that $\|T(u-\sigma)\|^{2}=\min _{s \in N S}\|T(u-s)\|^{2}$.

If there has another $\tilde{\sigma}$ satisfying the property too, that's mean $\|T(u-\tilde{\sigma})\|^{2}=\min _{s \in N S}\|T(u-s)\|^{2}$. Then when we take $u=\sigma, s=\tilde{\sigma}$, it is obvious that $\|T(\sigma-\tilde{\sigma})\|^{2}=0$. This means that $\sigma-\tilde{\sigma} \in N(T)$, thus $\sigma-\tilde{\sigma}=$ $\sum_{k=0}^{1} \sum_{l=0}^{1} c_{k l} x^{k} y^{l}$.

The other types have similar properties, and they also have similar proofs.

In the subsection, we apply the above constructed interpolation elements for scattered data.

Consider the function $u(x, y)=\cos x \times \sin y$. The boundary is $a=c=-1, b=d=5$. That is $R=[0,4] \times[0,4]$. Interpolatory points (scattered data) are produced by random functions belonging to $[0,4] \times[0,4]$. We fit the function $u$ by the bi-cubic natural spline interpolations for $\mathbf{E x 1 , 2 , 3 , 4}$.

Ex1. In order to compare with the respective interpolation effects of four types, we produce 3 sets of simple data: they are sparser near $x=b$ than near $x=a$ in the rectangular domain $R$. The interpolatory results for max errors and average errors are listed in Table 1 to Table 3, the interpolatory surfaces are plotted in Figure 1 to Figure 3.

The experimental results show that the effects of scattered data fitting in type III and in type IV are slightly better than the ones in type I and type II.

Table 1. The Error for 241 interpolatory knots.

\begin{tabular}{c|c|c|c|c}
\hline 241 interpolatory knots & type I & type II & type III & type IV \\
\hline max error & 0.026082 & 0.081083 & 0.008129 & 0.014179 \\
average error & 0.001110 & 0.001677 & 0.000666 & 0.000841 \\
\hline
\end{tabular}

Table 2. The Error for 481 interpolatory knots.

\begin{tabular}{c|c|c|c|c}
\hline 481 interpolatory knots & type I & type II & type III & type IV \\
\hline max error & 0.011998 & 0.013570 & 0.003512 & 0.005543 \\
average error & 0.000352 & 0.000447 & 0.000201 & 0.000206 \\
\hline
\end{tabular}

Table 3. The Error for 881 interpolatory knots.

\begin{tabular}{c|c|c|c|c}
\hline 881 interpolatory knots & type I & type II & type III & type IV \\
\hline max error & 0.001697 & 0.007026 & 0.000671 & 0.001980 \\
average error & $8.06967 \mathrm{E}-05$ & 0.000112 & $3.70628 \mathrm{E}-05$ & $5.54116 \mathrm{E}-05$ \\
\hline
\end{tabular}




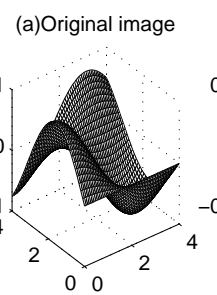

(b)Type I

(c)Type II

(d) distribution of knots
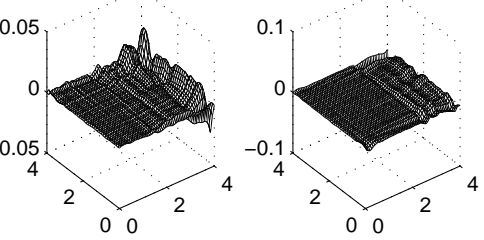

(e)Type III

(f)Type IV
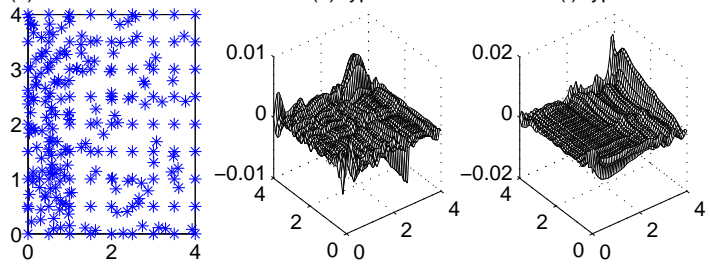

Figure 1. Interpolation results with different boundary conditions for 241 scattered data, (a)the image of the interpolated function(original image), (d)a distribution of 241 interpolatory knots, (b)its interpolant error with type I, (c)its interpolant error with type II, (e)its interpolant error with type III, (f)its interpolant error with type IV.

(a)Original image

(b)Type I

(c)Type II
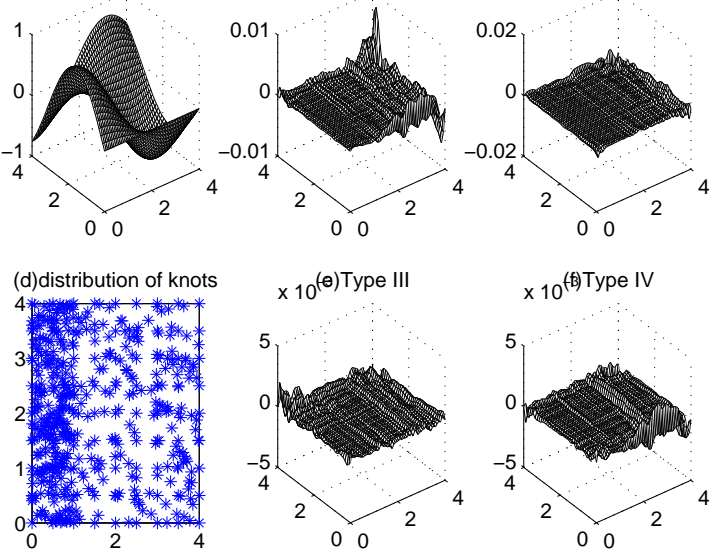

Figure 2. Interpolation results with different boundary conditions for 481 scattered data, (a)the image of the interpolated function(original image), (d)a distribution of 481 interpolatory knots, (b)its interpolant error with type I, (c)its interpolant error with type II, (e)its interpolant error with type III, (f)its interpolant error with type IV. 

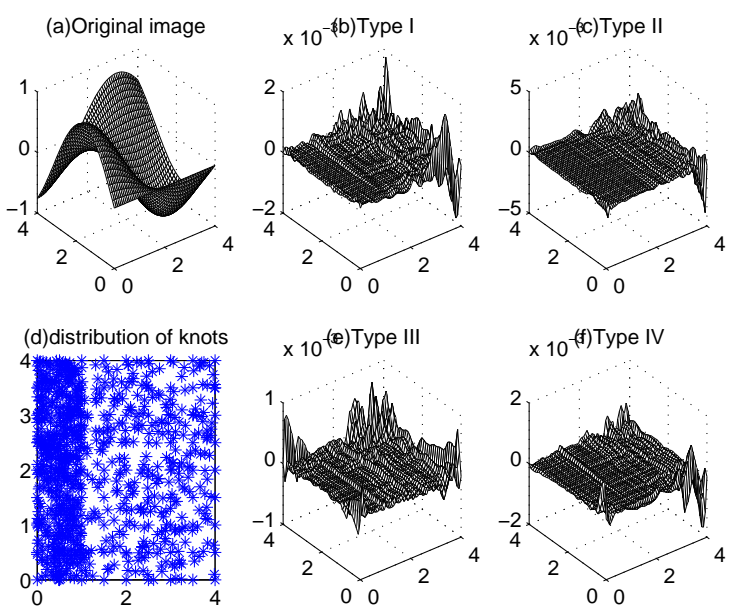

Figure 3. Interpolation results with different boundary conditions for 881 scattered data, (a)the image of the interpolated function(original image), (d)a distribution of 881 interpolatory knots, (b)its interpolant error with type I, (c)its interpolant error with type II, (e)its interpolant error with type III, (f)its interpolant error with type IV.

Ex2. In order to compare with the respective interpolation effects of four types, we produce 2 sets of simple data: they are sparser near the corner $x=b, y=d$ than the others in the rectangular domain $R$. The interpolatory results for max errors and average errors are listed in Table 4 and Table 5, the interpolatory surfaces are plotted in Figure 4 and Figure 5.

Table 4. The Error for 526 interpolatory knots.

\begin{tabular}{c|c|c|c|c}
\hline 526 interpolatory knots & type I & type II & type III & type IV \\
\hline max error & 0.009033 & 0.020292 & 0.003564 & 0.001504 \\
average error & 0.000214 & 0.000281 & 0.000173 & 0.000081 \\
\hline
\end{tabular}

Table 5. The Error for 731 interpolatory knots.

\begin{tabular}{c|c|c|c|c}
\hline 731 interpolatory knots & type I & type II & type III & type IV \\
\hline max error & 0.002046 & 0.007353 & 0.002856 & 0.000603 \\
average error & 0.000059 & 0.000142 & 0.000071 & 0.000032 \\
\hline
\end{tabular}

The experimental results show that the effects of scattered data fitting in type IV are slightly better than the ones in other types.

From Ex1 and Ex2, the interpolation errors for scattered data mainly include internal error and boundary error. The latter can be reduced by choosing the respective interpolation type when the data in one corner is very sparse. Moreover, for the general scattered data, the Partition of Unity Interpolation Element Method (PUIEM) is proposed.

\section{Partition of Unity Interpolation Element Method (PUIEM)}

We test our adaptivity on the partition of unity interpolation element method (PUIEM) for scattered data. The basic idea of the partition of unity method consists of decomposing the original domain into several subdomains or patches forming a covering of it, then constructing a local interpolation element approximant on each subdomain. Such an approach, as evident in our numerical analysis, enables us to significantly reduce the computational 

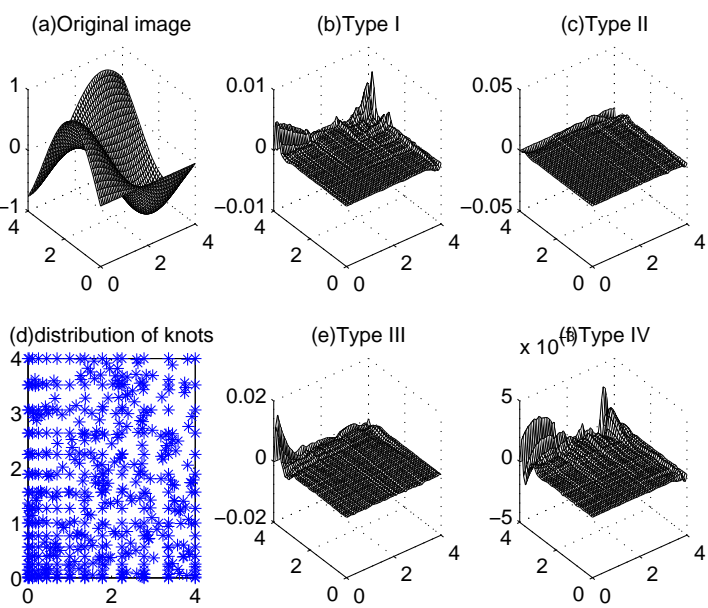

(e)Type III

$$
\times 10^{(\mathrm{B})} \text { Type IV }
$$
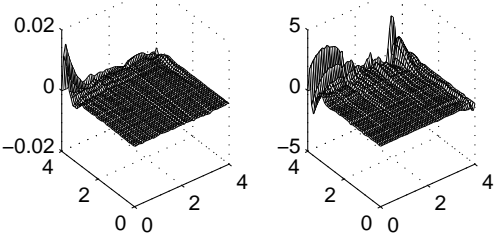

Figure 4. Interpolation results with different boundary conditions for 526 scattered data, (a)the image of the interpolated function(original image), (d)a distribution of 241 interpolatory knots, (b)its interpolant error with type I, (c)its interpolant error with type II, (e)its interpolant error with type III, (f)its interpolant error with type IV.
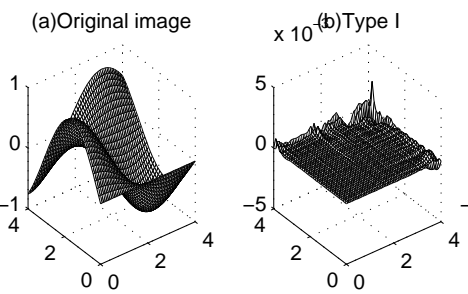

(c)Type II
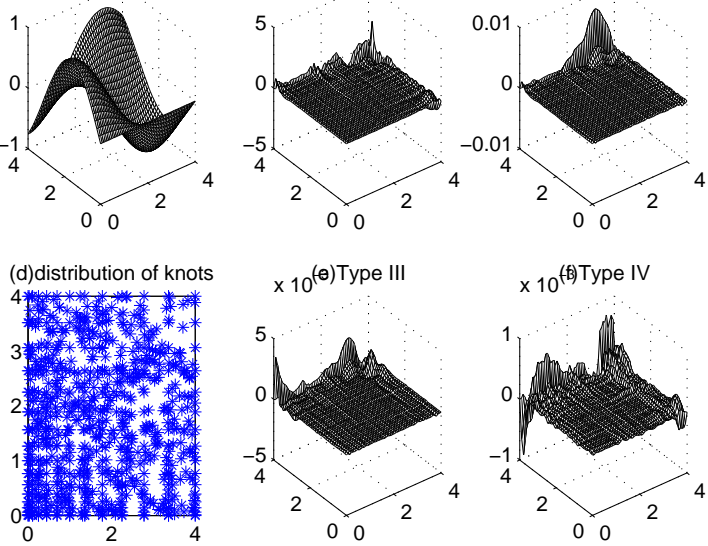

Figure 5. Interpolation results with different boundary conditions for 731 scattered data, (a)the image of the interpolated function(original image), (d)a distribution of 481 interpolatory knots, (b)its interpolant error with type I, (c)its interpolant error with type II, (e)its interpolant error with type III, (f)its interpolant error with type IV.

instability due to severe ill-conditioning generated by interpolation element methods. Originally, the partition of unity finite element method was introduced in $[14,15]$ to solve PDEs. Recently, this method has reached popularity because it allows one to efficiently and accurately solve large scale interpolation problems, as well as differential equation ones. However, the problem of constructing an adaptive method is still open and does not seem to have received the proper attention in literature. 
Partitioning $R$ into $k$ patches $\omega_{j}$ such that $\bigcup_{j=1}^{k} \omega_{j} \supseteq R$ with mild overlap among the patches, we can define the PUIEM by considering a partition of unity $\left\{\alpha_{j}\right\}_{j=1}^{k}$ subordinated to the covering $\left\{\omega_{j}\right\}_{j=1}^{k}$ such that

$$
\sum_{j=1}^{k} \alpha_{j}(x, y)=1, \forall(x, y) \in R
$$

The weight $\alpha_{j}: \omega_{j} \rightarrow \mathbb{R}$ is a compactly supported, nonnegative function with $\operatorname{supp}\left(\alpha_{j}\right) \subseteq \omega_{j}$. So the global PUIEM approximant assumes the form

$$
u(x, y) \approx f(x, y)=\sum_{j=1}^{k} \alpha_{j}(x, y) f_{j}(x, y),(x, y) \in R .
$$

The approximant $f_{j}$ is chosen from the bi-cubic natural spline interpolation elements with different boundary conditions.

Ex4. In order to compare with the respective interpolation effects of four types and PUIEM, we generate 621 random data in the rectangular domain $R$. For similarity, we consider the four small rectangular $\alpha_{j}, j=1,2,3,4$ which cover $R$ as shown in Figure 6. $f_{j}(x, y), j=1,2,3,4$ are the bicubic natural spline inpolation elements with corresponding boundary conditions.

The interpolatory results for max errors and average errors are listed in Table 6, the error surfaces of interpolation by four types and PUIEM are plotted in Figure 7.

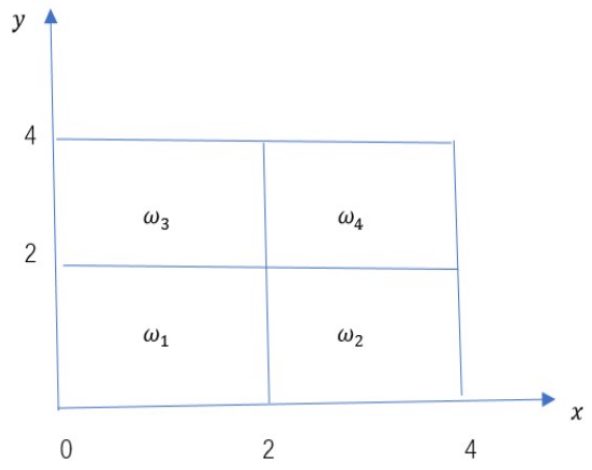

Figure 6. The partition of unity of domain $R$.

The experimental results show that the effects of scattered data fitting in PUIEM are better than four types.

Table 6. The Error for 621 interpolatory knots.

\begin{tabular}{c|c|c|c|c|c}
\hline 621 interpolatory knots & type I & type II & type III & type IV & PUIEM \\
\hline max error & 0.0129 & 0.0489 & 0.0158 & 0.0120 & 0.0073 \\
average error & 0.000221 & 0.000337 & 0.000165 & 0.000149 & 0.000122 \\
\hline
\end{tabular}

Ex5. In order to compare with the PUIEM and the thin plate spline $\left(\varphi(r)=r^{2} \ln |r|, r=\sqrt{\left(x-x_{i}\right)^{2}+\left(y-y_{i}\right)^{2}}\right)$ interpolation, we produce 2 sets of simple data: the gridded data and the scattered data. The latter are produced by random functions belong to $R=[0,1] \times[0,1]$. Denote $s d=\frac{N}{p \times q}$ the measure to scattered data, then $\frac{1}{N} \leq s d \leq 1$. 


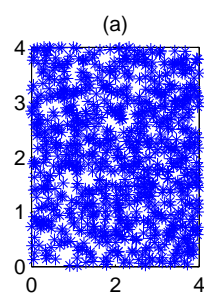

(d)

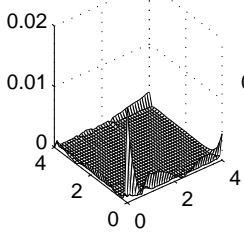

(b)

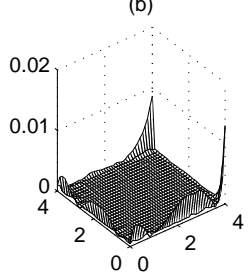

(e)

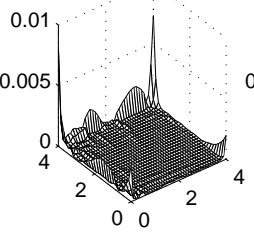

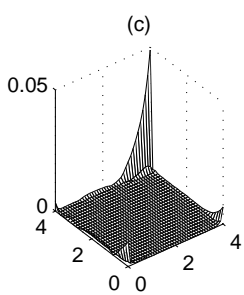

(f)

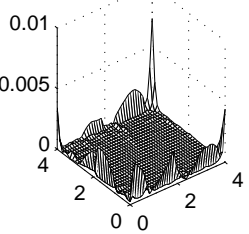

Figure 7. Interpolation results with different boundary conditions and PUIEM for 621 scattered data, (a)a distribution of 621 interpolatory knots, (b)its interpolant error with type I, (b)its interpolant error with type II, (c)its interpolant error with type III, (e)its interpolant error with type IV, (f)its interpolant error with PUIEM.

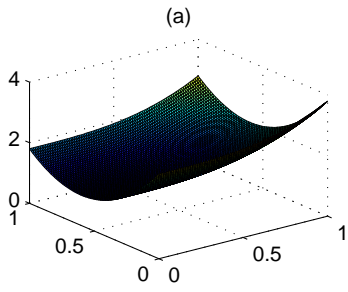

(c)

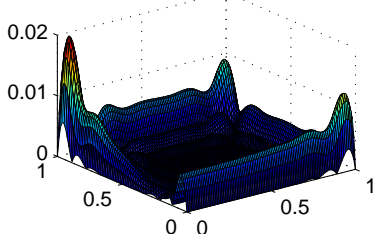

(b)

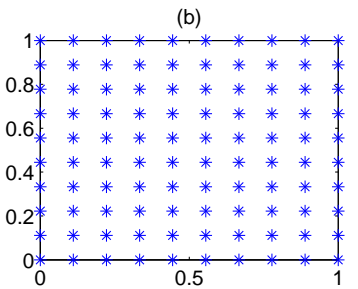

(d)

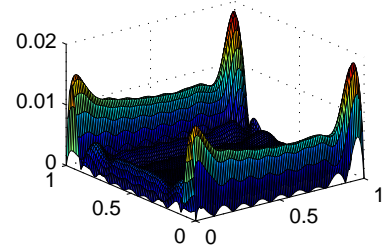

Figure 8. Interpolation results with the PUIEM and the thin plate spline(TPS) for 100 scattered data, (a)the image of the interpolated function(original image), (b)a distribution of 100 interpolatory knots, (c)its interpolant error with PUIEM, (d)its interpolant error with TPS.

Consider the function $u(x, y)=x^{3}+5(y-0.6)^{2}+1$. The boundary is $a=c=-1, b=d=2$. That is $R=$ $[0,1] \times[0,1]$. We fit the function $u$ by PUIEM and the thin plate spline(TPS) interpolation.

The interpolatory results for max errors and average errors are listed in Table 6 and Table 7, the interpolatory surfaces are plotted in Figure 7 and Figure 8.

Table 7. The Error for 100 interpolatory knots.

\begin{tabular}{c|c|c}
\hline$N=100, s d=1$ & average error & max error \\
\hline PUIEM & 0.001769 & 0.019929 \\
TPS & 0.002045 & 0.018976 \\
\hline
\end{tabular}


(a)

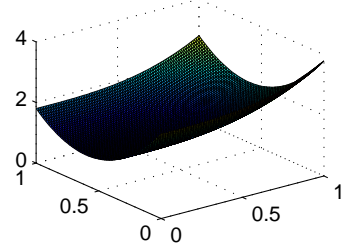

(c)

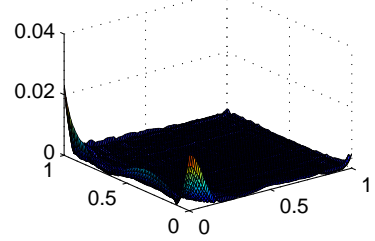

(b)

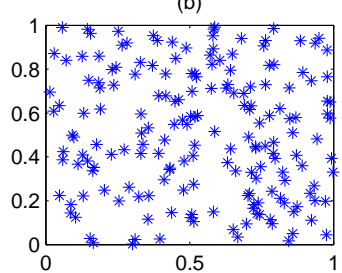

(d)

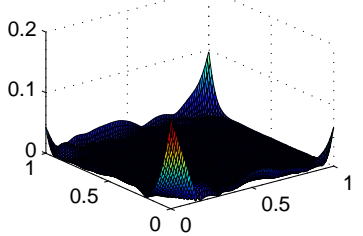

Figure 9. Interpolation results with the PUIEM and the thin plate spline(TPS) for 181 scattered data, (a)the image of the interpolated function(original image), (b)a distribution of 181 interpolatory knots, (c)its interpolant error with PUIEM, (d)its interpolant error with TPS.

Table 8. The Error for 181 interpolatory knots.

\begin{tabular}{c|c|c}
\hline$N=181, s d=0.0055$ & average error & max error \\
\hline PUIEM & 0.000494 & 0.023206 \\
TPS & 0.002688 & 0.147866 \\
\hline
\end{tabular}

Ex6. The function $u(x, y)=x^{3}+5(y-0.6)^{2}+1$ on $R=[0,1] \times[0,1]$. The experimental results in Figure 9 show that the effects of scattered data fitting in the PUIEM are better than the ones in the thin plate spline.

In conclusion, the experimental results show that Our PUIEM is adaptive and superior to the four natural spline interpolation types and the thin plate spline in the interpolation results.

\section{Conclusion}

In this paper, One kind of interpolation for scattered data by bi-cubic polynomial natural spline with different boundary conditions is studied. By the spline function methods of Hilbert space, their solutions are constructed as the sum of bi-linear polynomials and piecewise bi-cubic polynomials. Many properties of the solutions are studied and it is concluded that bi-cubic natural spline interpolation on a rectangular domain is a generalization of the cubic natural spline interpolation on an interval. Moreover, Based on partition of unity technique and bi-cubic polynomial natural spline interpolations of four kinds of boundary conditions, Partition of Unity Interpolation Element Method (PUIEM) for fitting scattered data is proposed. The experiments show that the PUIEM is adaptive and outperforms the bi-cubic polynomial natural spline interpolations of four kinds and the thin plate spline in the interpolation results for scattered data.

In a general way, the effects of scattered data fitting depend mainly on their distribution (e.g., sparsity and irregular boundaries). When the data in one corner is very sparse, it is suggested to choose the PUIEM or the respective bicubic natural spline interpolation type to minimize the error. Of course, it is also suggested that to choose more interpolatory points uniformly for data collection. In the future, the refined partition of unity will be considered and the large scale real scattered data (e.g., geographical data [18]) will be tested. 


\section{Acknowledgement}

The author wants to express his thanks to Prof. Lvtai Guan for his suggestions and comments. This work has been partly supported by the supported by the Natural Science Foundation of China under Grants 11701162 .

\section{REFERENCES}

1. J. Ahlberg, E. Nilson and J. Walsh, The Theory of Splines and Their Applications, Academic Press, New York, 1967.

2. C. de Boor, A Practical Guide to Splines, Springer Verlag Publishers, 2001.

3. S. Kersey and M. Lai, Convergence of Local Variational Spline Interpolation, Journal of Computational Analysis and Applications, 341: 398-415, 2008.

4. R. Wang, Multivariate Spline Functions and Their Applications, Kluwer Academic Publishers, The Netherlands, 2001.

5. G. Nurnberger and F. Zeilfelder, Developments in bivariate spline interpolation, Journal of Computational and Applied Mathematics, 121:125-152, 2000.

6. M. Lai and L. Schumaker, Spline Functions Over Triangulations, London: Cambridge University Press, 2007.

7. T. Zhou, D. Han and M. Lai, Energy Minimization Method for Scattered Data Hermit Interpolation, Applied Numerical Mathematics, 58: 646-659,2008.

8. M. Lai, Multivarariate Splines for Data Fitting and Approximation, Approximation Theory XII, San Antonio, 2007, edited by M. Neamtu and L.L.Schumaker, Brentwood: Nashboro Press, 2008: 210-228, 2008.

9. L. Guan and Y. Li, Multivariate polynomial natural spline interpolation to scattered data, Academic Press, New York, 1989.

10. C. Chui and L. Guan, Multivariate polynomial natural splines for interpolation of scattered data and other applications, World Scientific Pub, Singapore, 1993.

11. L. Guan and B. Liu, Surface Design by Natural Splines Over Refined Grid Points, Journal of Computational and Applied Mathematics, 163(1):107-115, 2004

12. A. Bezhaev and V. Vasilenko, Variational Theory of Splines, Kluwer Academic/Plenum Publishers, New York, 2001.

13. L. Guan, W. Xu and Q. Zhu, Interpolation for Space Scattered Data by Bicubic Polynomial Natural Splines, Acta Scientiarum Naturalium Universitatis Sunyatseni, 47(5): 1-4, 2008.

14. J. Melenk and I. Babuka, The partition of unity finite element method: basic theory and applications, Computer Methods in Applied Mechanics and Engineering, 139: 289-314, 1996.

15. I. Babuka, U. Banerjee and J. Osborn, Generalized finite element methods: main ideas, result and perspective, International Journal of Computational Methods, 1(1): 67-103, 2004.

16. K. Segeth, Some splines produced by smooth interpolation, Applied Mathematics and Computation, 319: 387-394, 2018.

17. H. Johnson and M. Johnson, Quasi-elastic cubic splines in $\mathbb{R}^{d}$, Computer Aided Geometric Design, 81: 101893, 2020.

18. K. Gao, G. Mei, S. Cuomo, F. Piccialli and N. Xiong, Adaptive RBF interpolation for estimating missing values in geographical data, Available at http://arxiv.org/abs/1908.03690, 2019. 